\title{
Stem Cells: Epigenetic Basis of Differentiation
}

\author{
Michael D. Williams ${ }^{1}$, Geraldine M. Mitchell ${ }^{1}$ and Anandwardhan A. Hardikar ${ }^{*}, 1,2$ \\ ${ }^{I}$ O'Brien Institute, 42 Fitzroy Street, VIC 3065, Australia and The University of Melbourne, Department of Surgery at St \\ Vincent's Hospital Melbourne, VIC 3065, Australia \\ ${ }^{2}$ Diabetes and Stem Cell Section, National Center for Cell Science, Ganeshkhind Road, Pune 411007, India
}

\begin{abstract}
Generation of mature differentiated cells is essential for the treatment of several diseases that depend on cell replacement therapies. With the growing knowledge of transcriptome diversity in cells, increased understanding now exists on the potential to inter-convert a specialized cell type into a differentiated cell of another lineage (transdifferentiation). Transcription in terminally committed cells is controlled by many extracellular and intracellular components and the intrinsic structure and confirmation of the DNA itself (the epigenome). The patterns of these modifications in differentiated cells are generally stable and heritable with characteristic modification patterns reflecting the phenotype they acquire during differentiation. Adult tissue-derived stem or progenitor cells possess inherent traits that result in "commitment" to a particular phenotype, demonstrated by their relatively restricted differentiation capacity. Adult tissue-derived stem cell populations represent a source of cells that would predictably require fewer manipulations to achieve an alternative, differentiated phenotype. By characterising cells with respect to epigenetic patterns, it may be possible to identify stem / progenitor cells that are poised to differentiate towards a particular lineage. Assessing the chromatin compactness at gene promoter regions may assist in identifying mechanisms for inducing cells to adopt a specific phenotype with increased efficiency of differentiation.
\end{abstract}

Keywords: Stem cells, epigenetic, trans-differentiation, transcriptome, epigenome.

\section{INTRODUCTION}

Diseases such as diabetes, multiple sclerosis, muscular dystrophy and myocardial infarction cannot be completely cured with conventional therapies. Regenerative medicine using alternative stem cell therapies may provide a potential biological solution to address such conditions. Regenerative medicine represents a mixed medical discipline, combining collective knowledge of physiology, cell biology, genetics and engineering to apply stem cells, biomaterial scaffolds and cytokine growth factors for treatment of several diseases, such as type 1 diabetes mellitus.

It is well known that human cells are diverse with respect to their epigenome, transcriptome and proteome. However, all cells are derived from a single cell; the fertilized ovum. The concept of dedifferentiation of cells back to a precursor or stem-like cell type and then inter-conversion to another type of differentiated cell (transdifferentiation) has been widely discussed [1-5]. Utilizing the process of transdifferentiation to provide cells for the treatment of several diseases including diabetes has enormous practical potential to treat common diseases which inflict millions of sufferers world-wide.

Type 1 Diabetes is caused by loss of insulin-producing beta cells in the pancreatic Islet of Langerhans. Although significant advances in treating diabetes were made in the

*Address correspondence to this author at the O’Brien Institute, and The University of Melbourne, Department of Surgery at St Vincent's Hospital, 42 Fitzroy Street, VIC 3065, Australia; Fax: +61 (3) 9416 0926; E-mail: anandh@unimelb.edu.au past few decades, lack of suitable organ donors for islet or beta cell transplantation is still a major hurdle. An alternative approach to overcome donor islet shortage is to produce beta cells in the laboratory for transplantation into patients. Research from our group and others demonstrates the possibility of inter-conversion of cells harvested from the gallbladder or liver into insulin-producing cells [6-8]. However, none of the studies published to date have been successful in producing cells identical to islet beta cells. Generating lineage-committed islet progenitor cells is a major challenge in cell replacement therapy for diabetes.

In the past decade, the term "stem cell" has been loosely defined by the capacity for extensive self-renewal and provision of abundant daughter cells. However, research in understanding the programming of stem cells to differentiate into a single specialized tissue, or organ specific cell type by acquiring the correct phenotypic / biochemical and functional properties remains to be explored. Stem cells are typically classified into one of three classes: embryonic, postnatal and induced pluripotent stem (iPS) cells. Embryonic stem (ES) cells are isolated from the inner cell mass and considered to be pluripotent, with the potential to differrentiate into almost all the tissues/organs that arise from the three germ layers (however these cells cannot form a complete embryo, lacking the capacity to form placental and supporting tissues) $[9,10]$. The choice of embryonic stem cells for potential cell therapies is highly debated and remains controversial [11]. Post-natal (adult / progenitor) stem cells have been identified in virtually all organs in the body and are thought to represent reservoirs of precursor cells that contribute to maintenance and regeneration of the organ / tissue throughout life. These cells tend to 
demonstrate a limited differentiation potential, generally restricted to forming differentiated cell types in the tissue from which they are derived $[9,10]$. The division between embryonic and post-natal stem cells has become blurred following the demonstration of adult murine fibroblasts reprogrammed to pluripotency though the application of four factors (Oct3/4, Sox2, c-Myc, and Klf4, under embryonic stem cell culture conditions) to generate induced pluripotent stem (iPS) cells [12]. These iPS cells were injected into blastocysts and shown to contribute to all three germ layers. These findings questioned the term "stemness", which was thought to be only a property of embryonic stem cells. The term "stemness" can now be defined as a property of the cell; the property of having a specific set of genes whose expression allows potential for self-renewal as well as for multilineage differentiation and regeneration. It has been suggested that stemness may represent a transient and reversible trait that is acquired by cells when exposed to the necessary environmental cues (niche) [13]. The resulting expression and localization of proteins, nucleic acids, lipid and specific patterns of chromatin organization give rise to a stem-cell phenotype. Two alternative models of stem cell differentiation have been proposed [13, 14]. The first assumes that the stem cells represent a "blank slate" and do not express any genes characteristic of differentiation. Upon induction to differentiate, cells acquire specific differentiation markers in a step-by-step manner until they achieve a mature phenotype or in other words, "commit" to a specific lineage. The second model proposes that stem cells express very low levels of "specialized" markers. Differentiation is characterized by an increase in expression of these markers characteristic of the lineage accompanied by simultaneous silencing of gene expression for unrelated lineages.

\section{EPIGENETIC REGULATION OF STEM CELL TRANSCRIPTOME}

Transcription in terminally committed cells is controlled by many extracellular and intracellular components and the intrinsic structure and confirmation of the DNA itself (the epigenome). In eukaryotic cells, DNA exists as chromatin, composed of the DNA and associated nuclear proteins; histones. Each repeating unit (nucleosome) is composed of an octameric protein core containing two subunits of $\mathrm{H} 2 \mathrm{~A}$, $\mathrm{H} 2 \mathrm{~B}, \mathrm{H} 3$ and $\mathrm{H} 4$ and a 146 base pair DNA wrapped around this histone octamer $[15,16]$. Histone interactions are mediated through the phosphodiester skeleton of the DNA molecule and as such are largely independent of the DNA sequence. Histones undergo several different post-translational modifications including acetylation, methylation, phosphorylation, sumoylation and ubiquitination. DNA methylation also represents heritable epigenetic marks for active or inactive chromatin [17]. DNA methylation of cytosine in $\mathrm{CpG}$ dinucleotides is mediated by DNA methyltransferases [18-20]. DNA methyltransferase 1 (Dnmt1) is a maintenance enzyme that methylates hemi-methylated $\mathrm{CpG}$ dinucleotides to preserve methylation patterns in proliferating cells. Dnmt3a and Dnmt3b are responsible for methylation patterning of the DNA in development and have been shown to interact with histone modifying enzymes to repress transcription. DNA methylation is typically associated with persistent gene silencing [21]. Histone modification is mediated through covalent modifications of the exposed $\mathrm{N}$ terminal tails of the histone amino acid chains [22]. Multiple modification mechanisms have been characterized including acetylation of the lysine resides, methylation of lysine and arginine residues [20,23], phosphorylation of serine residues [24], poly(ADP) ribosylation of glutamate residues [25], ubiquitinylation and sumoylation [26].

Transcriptionally active chromatin contains characteristic site-specific modifications to the N-terminal domains of histone molecules. Acetylation/deacetylation of lysines correlates with chromatin accessibility and transcription, whereas the effect of lysine methylation depends on the number of methyl groups and position of lysine residues. The less compacted nature of euchromatin leaves DNA open and accessible to facilitate transcription. Trimethylation of histone H3 lysine 4 (H3K4-me3) and acetylation of H3 and $\mathrm{H} 4$ are associated with active transcription [27, 28]. High levels of acetylation and trimethylation of $\mathrm{H} 3 \mathrm{~K} 4, \mathrm{H} 3 \mathrm{~K} 36$, and $\mathrm{H} 3 \mathrm{~K} 79$ is synonymous with transcriptionally active genes while low levels of these modifications are associated with inactive genes. How these modifications manifest as altered transcription activity is not fully understood, however trimethylation of $\mathrm{H} 3 \mathrm{~K} 4$ is thought to mediate recruitment of chromatin-remodelling complexes to promoters [29], with long non-coding (nc)RNAs possibly acting as a modular scaffold for histone modification complexes [30].

The patterns of these modifications in differentiated somatic cells are generally stable and heritable $[19,31]$ with characteristic modification patterns reflecting the phenotype they acquire during differentiation. Cells obtained from the early blastula have revealed that the DNA of a newly created embryo is highly unmethylated [32], which persists until implantation [33]. As cells begin to commit to specific lineages, they acquire a specific phenotype dictated by tissue-specific transcription factors and develop characteristic epigenetic modifications. Genes associated with pluripotency become inaccessible to transcription factors with remodelling of chromatin and are therefore silenced. Conversely, the chromatin in the region of genes associated with a particular cell fate or lineage achieves an "open" conformation so as to provide access for the binding of transcriptional machinery. During embryonic development, the entire genome is known to go through several levels of compaction and relaxation of chromatin. This process is initiated through recruitment of specific enzymes that engage in post-translational modification of histones. The developing tissues then achieve specific phenotypes based on spatio-temporal expression of factors that can be collectively termed as growth and differentiation factors (GDFs) [34]. Pancreatic stem / progenitor cells are exposed to different concentrations of these GDFs during embryonic development to give rise to differentiated insulin-producing cells in the Islets of Langerhans. Such differentiated cells carry the epigenetic marks that allow open chromatin conformation at the insulin promoter region as well as the key pancreatic transcription factors (such as Pdx1, MafA) in islet beta cells [35]. Therefore adult tissue-derived stem or progenitor cells possess inherent traits that result in a predisposition or "commitment" to a particular phenotype, demonstrated by their relatively restricted differentiation capacity as compared to other cell types, such as ES cells. These populations represent a source of cells that would predictably require 
Transcript abundance

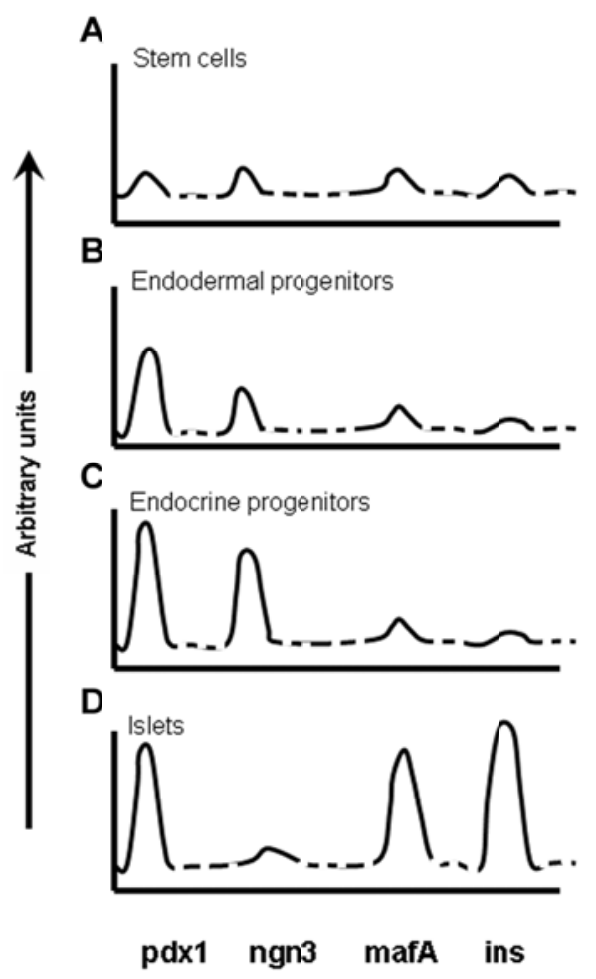

Chromatin conformation

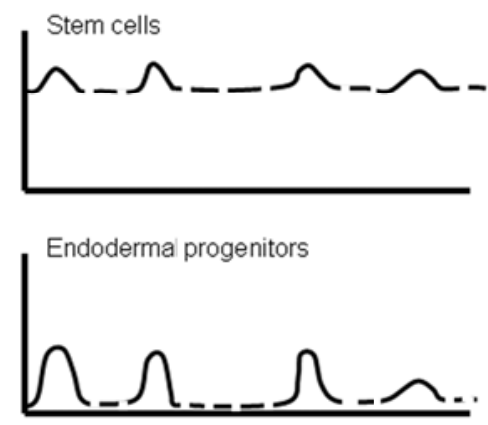

Endocrine progenitors
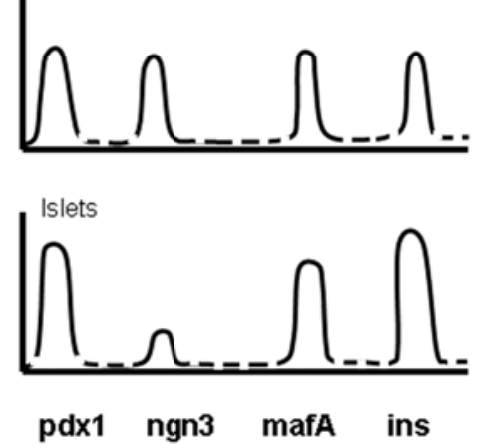

Fig. (1). A representation of transcript abundance and chromatin conformation of stem and islet progenitor cells. (A) Stem cells have a relatively open chromatin conformation and therefore demonstrate expression of endocrine progenitor markers albeit at lower levels. With successive differentiation into endodermal (B) and pancreatic (C) lineages, the accessible chromatin (seen in panel A) is replaced by selective silencing of specific genes. Islets (D) represent a highly efficient insulin-producing cell type that offers a suitable chromatin conformation, which allows the transcriptional machinery to bind to the promoter region and transcribe these genes. An optimal promoter conformation is the major key to efficient transcription / differentiation of cells.

fewer manipulations than pluripotent cells to achieve their differentiated phenotype [36]. Although the specific mechanisms responsible for the intrinsic propensity of cells to differentiate along a particular lineage remain largely unknown, we theorize that epigenetic mechanisms, including DNA methylation, covalent histone modifications, and noncoding RNA-mediated gene regulation are largely involved in development of a differentiated phenotype [37, 38] (see Fig. 1).

Gene expression is also epigenetically regulated at the posttranscriptional level through an additional epigenetic mechanism; non-coding microRNAs (miRNAs). miRNAs represent an evolutionary conserved class of short (18-22 bp) non-protein-coding RNA molecules. They can be classified into three categories $[39,40]$ based on their location in the genome; (i) exonic miRNAs in non-coding gene transcripts, (ii) intronic miRNAs in non-coding gene transcripts and (iii) intronic miRNAs in protein-coding gene transcripts. miRNAs mediate the regulation of gene expression based on the level of complementarity between the given miRNA seed sequence (first 2 to $8 \mathrm{bp}$ of miRNA) and regulatory regions, such as RNA interference and RNA editing [41]. miRNAs are known to negatively regulate gene expression at the posttranscriptional level, either by inhibiting translation or by degrading the target mRNA $[42,43]$. Several miRNAs (miR- 15a, miR-15b, miR-16 and miR-195) have been identified as targeting ngn3 during pancreatic regeneration, implicating these miRNAs in the regulation of islet beta-cell regeneration $[40,44]$. This understanding will potentially yield a novel targeted manipulation of these specific miRNAs for the induction of differentiation of pancreatic progenitor populations and possibly other less related cell populations.

The coming years will uncover the potential regulatory control of these iRNAs in cell differentiation.

\section{EPIGENETICS AND STEM CELL BIOLOGY}

Adult or progenitor cells inherit epigenetic modifications following proliferation. Therefore, by characterising cells with respect to epigenetic patterns, it may be possible to identify cells that are poised to differentiate towards a particular lineage. By assessing the compactness of chromatin at specific gene promoter regions that are located with regions of transcriptionally permissive chromatin, it is possible to identify methods of inducing the cells to adopt a specific phenotype. Current methods of altering the epigenome of isolated cells involve the use of DNA methyltransferases and histone deacetylase inhibitors which act on the epigenome on a global scale [45]. These agents are largely non-specific, preventing the targeted application to chromosomal regions 
or gene promoters to elicit specific effects. The application of these agents is therefore limited, however they have been shown to alter cell differentiation [23, 46, 47] and their applications in anticancer therapy is being explored [45, 48], with DNA methyltransferase inhibitors like 5-azacytidine and 5-aza-2'-deoxycytidine currently FDA approved for the treatment of myelodysplastic syndrome.

The advantage of using adult stem cells over more pluripotent cell sources, such as embryonic stem cells, is their apparently restricted potential to differentiate into specific lineages, which is inherited in their epigenome. As stated previously, the predisposition for adult stem cells to differentiate into a particular cell type means that these cells would predictably require less manipulation to achieve the desired cell type. Compared to adult stem cells, embryonic stem cells are thought to require more extensive manipulation to direct them towards a specific lineage [36]. The open chromatin conformation seen at multiple promoter regions in ES cells also suggests the rationale for open chromatin structure in stem cells. Adults stem cells are also a potentially safer cell source, compared to embryonic stem cells, which are known to form teratomas, a specific type of tumour consisting of derivatives of all three germ layers when implanted into ectopic sites $[49,50]$. This effect has not been reported following implantation of adult stem (progenitor) cells. Exploration of the clinical potential of adult stem cells in regenerative medicine provides a potentially large pool of cells, which are ethically far less contentious than embryonic stem cells. However, the collective efforts to characterize and understand developmental cues for patterning and differentiation of cells throughout development, embryonic and adult, are vital to understanding and predicting the behaviour and activity of adult stem cells. However, the future of regenerative medicine lies with adult or progenitor stem cells opposed to embryonic stem cells, at least in the foreseeable future.

\section{ADULT STEM CELLS FOR REGENERATIVE MEDICINE}

The governing criteria for the choice of adult stem cells in pre-clinical and clinical studies has included the availability and expansion of isolated cells in accordance with current Good Manufacturing Practice, their potential to be reliably directed along a particular lineage and their safe application in a clinical setting [51]. In accordance with these characteristics mesenchymal cells isolated from the bone marrow, adipose tissue and peripheral blood and umbilical cord-derived mononuclear cells may represent potential candidates for therapeutic applications in a clinical setting. Cells obtained from bone marrow and adipose tissue have demonstrated multilineage potential and promise of applications in regenerative medicine $[10,52-54]$, as have peripheral blood [55-57] and umbilical cord [58-60] cells. However, the epigenome of these respective cell populations remains largely undefined [61]. The choice and implementtation of cells for the regenerative treatment of patients suffering with type 1 diabetes mellitus is of particular interest to our group. For a treatment to be successful, a sufficient number of insulin-producing cells must be implanted into the patient, such that the individual can maintain normal circulating glucose concentrations. Allogenic transplantation of the Islets of Langerhans from human cadavers [62] has been approved for clinical research and has achieved positive results. However, this technology is limited by the low number of cadaveric donors and the restricted yield of viable cells for transplantation. Therefore, research groups around the world are seeking alternative cell sources with the potential to give rise to insulin-expressing cells for transplantation $[63,64]$. In line with our hypothesis regarding the use of differentiated cell types, we believe that cells isolated from pancreatic tissues (endocrine, acinar or ductal) or cells isolated from other (particularly endodermal) tissues (gallbladder and liver) represent alternative cell sources for reliable generation of insulin-producing cells. There is now a growing body of evidence to suggest that it is possible to induce the transdifferentiation of hepatocytes [6, $65,66]$, intra-/extrahepatic biliary epithelial cells [7] and gall-bladder epithelium [8] to express markers characteristic of the pancreatic lineage with relatively little in vitro manipulation. These studies demonstrate the potential of adult stem cells from a range of tissue sources to be directed to achieve an endocrine pancreas phenotype, and the optimization of these techniques for use in clinical applications is currently under investigation.

\section{CONCLUSION}

We are now only beginning to understand the roles of epigenetic characteristics and non-coding RNAs in cell behaviour and the maintenance of specific phenotypes. This may have significant implications for the choice of adult stem cells for clinical applications and the techniques used to achieve differentiation to the desired lineage. By combining this knowledge with the information already acquired about the differentiation of cells in culture, future studies will identify novel regulatory pathways and in vitro methods capable of achieving robust and reliable differentiation of isolated adult stem cells for downstream applications in a clinical scenario.

\section{ACKNOWLEDGEMENTS}

Michael Williams is a $\mathrm{PhD}$ student and recipient of an Australian Postgraduate Award. The O'Brien Institute acknowledges the Victorian State Government's Department of Innovation, Industry and Regional Development's Operational Infastructure Support Program.

\section{REFERENCES}

[1] Barzilay R, Melamed E, Offen D. Introducing transcription factors to multipotent mesenchymal stem cells: making transdifferentiation possible. Stem Cells 2009; 27(10): 2509-15.

[2] Reinecke H, Minami E, Zhu W-Z, et al. Cardiogenic differentiation and transdifferentiation of progenitor cells. Circ Res 2008; 103(10): 1058-71.

[3] Zhou Q, Melton DA. Extreme makeover: converting one cell into another. Cell Stem Cell 2008; 3(4): 382-8.

[4] Kordowich S, Mansouri A, Collombat P. Reprogramming into pancreatic endocrine cells based on developmental cues. Mol Cell Endocrinol 2010; 323(1): 62-9.

[5] Minami K, Seino S. Pancreatic acinar-to-beta cell transdifferentiation in vitro. Front Biosci 2008; 13: 5824-37.

[6] Ferber S, Halkin A, Cohen H, et al. Pancreatic and duodenal homeobox gene 1 induces expression of insulin genes in liver and 
ameliorates streptozotocin-induced hyperglycemia. Nat Med 2000. 6(5): p. 568-72.

[7] Nagaya M. Adult mouse intrahepatic biliary epithelial cells induced in vitro to become insulin-producing cells. J Endocrinol 2009; 201(1): 37-47.

[8] Sahu S. Islet-like cell clusters occur naturally in human gall bladder and are retained in diabetic conditions. J Cell Mol Med 2009; 13(5): 999-1000.

[9] Alison MR, Islam S. Attributes of adult stem cells. J Pathol 2009; 217(2): 144-60.

[10] da Silva Meirelles L, Chagastelles PC, Nardi NB. Mesenchymal stem cells reside in virtually all post-natal organs and tissues. J Cell Sci 2006; 119(Pt 11): 2204-13.

[11] Lo B, Parham L. Ethical issues in stem cell research. Endocr Rev 2009; 30(3): 204-13.

[12] Takahashi K, Yamanaka S. Induction of pluripotent stem cells from mouse embryonic and adult fibroblast cultures by defined factors. Cell 2006; 126(4): 663-76.

[13] Zipori D. The nature of stem cells: state rather than entity. Nat Rev Genet 2004; 5(11): 873-8.

[14] Delorme B, Ringe J, Pontikoglou C, et al. Specific lineage-priming of bone marrow mesenchymal stem cells provides the molecular framework for their plasticity. Stem Cells 2009; 27(5): 1142-51.

[15] Luger K, Hansen JC. Nucleosome and chromatin fiber dynamics. Curr Opin Struct Biol 2005; 15(2): 188-96.

[16] Razin SV, Iarovaia OV, Sjakste N, et al. Chromatin domains and regulation of transcription. J Mol Biol 2007; 369(3): 597-607.

[17] Li E. Chromatin modification and epigenetic reprogramming in mammalian development. Nat Rev Genet 2002; 3(9): 662-73.

[18] Goll MG, Bestor TH. Eukaryotic cytosine methyltransferases. Annu Rev Biochem 2005; 74: 481-514.

[19] Bird A. DNA methylation patterns and epigenetic memory. Genes Dev 2002; 16(1): 6-21.

[20] Barski A. High-resolution profiling of histone methylations in the human genome. Cell 2007; 129(4): 823-37.

[21] Reik W. Stability and flexibility of epigenetic gene regulation in mammalian development. Nature 2007; 447(7143): 425-32.

[22] Davey CA, Sargent DF, Luger K, et al. Solvent mediated interactions in the structure of the nucleosome core particle at $1.9 \mathrm{a}$ resolution. J Mol Biol 2002; 319(5): 1097-113.

[23] Wang G, Badylak SF, Heber-Katz E, et al. The effects of DNA methyltransferase inhibitors and histone deacetylase inhibitors on digit regeneration in mice. Regen Med 2010; 5(2): 201-20.

[24] Zheng Y, John S, Pesavento JJ, et al. Histone H1 phosphorylation is associated with transcription by RNA polymerases I and II. J Cell Biol 2010; 189(3): 407-15.

[25] Malik N, Sugimura T, Thraves $\mathrm{P}$, et al. Immunoaffinity fractionation of the poly(ADP-ribosyl)ated domains of chromatin. Proc Natl Acad Sci USA 1983; 80(9): 2554-8.

[26] Shiio Y, Eisenman RN. Histone sumoylation is associated with transcriptional repression. Proc Natl Acad Sci USA 2003; 100(23): 13225-30.

[27] Bernstein BE, Kamal M, Lindblad-Toh K, et al. Genomic maps and comparative analysis of histone modifications in human and mouse. Cell 2005; 120(2): 169-81.

[28] Kouzarides T. Chromatin modifications and their function. Cell 2007; 128(4): 693-705.

[29] Wysocka J, Swigut T, Xiao H, et al. A PHD finger of NURF couples histone $\mathrm{H} 3$ lysine 4 trimethylation with chromatin remodelling. Nature 2006; 442(7098): 86-90.

[30] Tsai MC, Manor O, Wan Y, et al. Long noncoding RNA as modular scaffold of histone modification complexes. Science 2010; 329(5992): 689-93.

[31] McNairn AJ, Gilbert DM. Epigenomic replication: linking epigenetics to DNA replication. Bioessays 2003; 25(7): 647-56.

[32] Kafri T, Ariel M, Brandeis M, et al. Developmental pattern of genespecific DNA methylation in the mouse embryo and germ line. Genes Dev 1992; 6(5): 705-14.

[33] Okano M, Bell DW, Haber DA, et al. DNA methyltransferases Dnmt3a and Dnmt3b are essential for de novo methylation and mammalian development. Cell 1999; 99(3): 247-57.

[34] Tsuchida K. Activins, myostatin and related TGF-beta family members as novel therapeutic targets for endocrine, metabolic and immune disorders. Curr Drug Targets Immune Endocr Metabol Disord 2004; 4(2): 157-66.
[35] Gaulton KJ, Nammo A, Pasquali L, et al. A map of open chromatin in human pancreatic islets. Nat Genet 2010; 42(3): 255-9.

[36] Gershengorn MC, Geras-Raaka E, Hardikar AA, et al. Are better islet cell precursors generated by epithelial-to-mesenchymal transition? Cell Cycle 2005; 4(3): 380-2.

[37] Cho KS, Elizondo LI, Boerkoel CF. Advances in chromatin remodeling and human disease. Curr Opin Genet Dev 2004; 14(3): 308-15.

[38] Dalvi MP, Umrani MR, Joglekar MV, et al. Human pancreatic islet progenitor cells demonstrate phenotypic plasticity in vitro. J Biosci 2009; 34(4): 523-8

[39] Kim VN. MicroRNA biogenesis: coordinated cropping and dicing. Nat Rev Mol Cell Biol 2005; 6(5): 376-85.

[40] Joglekar MV, Parekh VS, Hardikar AA. New pancreas from old: microregulators of pancreas regeneration. Trends Endocrinol Metab 2007; 18(10): 393-400.

[41] Zaratiegui M, Irvine DV, Martienssen RA. Noncoding RNAs and gene silencing. Cell 2007; 128(4): 763-76.

[42] Ying SY, Chang DC, Lin S-L, et al. The microRNA: overview of the RNA gene that modulates gene functions. Methods Mol Biol 2006; 342: 1-18.

[43] Tanzer A, Stadler PF. Evolution of microRNAs. Methods Mol Biol 2006; 342: 335-50.

[44] Joglekar MV, Parekh VS, Mehta S, et al. MicroRNA profiling of developing and regenerating pancreas reveal post-transcriptional regulation of neurogenin3. Dev Biol 2007; 311(2): 603-12.

[45] Fandy TE. Development of DNA methyltransferase inhibitors for the treatment of neoplastic diseases. Curr Med Chem 2009; 16(17): 2075-85.

[46] Mahpatra S, Firpo MT, Bacanamwo M. Inhibition of DNA methyltransferases and histone deacetylases induces bone marrowderived multipotent adult progenitor cells to differentiate into endothelial cells. Ethn Dis 2010; 20(1 Suppl 1): S1-60-4.

[47] Choi YS, Dusting GJ, Stubbs S, et al. Differentiation of human adipose-derived stem cells into beating cardiomyocytes. J Cell Mol Med 2010; 14(4): 878-89.

[48] Sebova K, Fridrichova I. Epigenetic tools in potential anticancer therapy. Anticancer Drugs 2010; 21(6): 565-77.

[49] Molcanyi M, Riess P, Haj-Yasein NN, et al. Developmental potential of the murine embryonic stem cells transplanted into the healthy rat brain--novel insights into tumorigenesis. Cell Physiol Biochem 2009; 24(1-2): 87-94.

[50] Denker HW. Potentiality of embryonic stem cells: an ethical problem even with alternative stem cell sources. J Med Ethics 2006; 32(11): 665-71.

[51] Gimble JM, Katz AJ, Bunnell BA. Adipose-derived stem cells for regenerative medicine. Circ Res 2007, 100(9): 1249-60.

[52] Pittenger MF, Mackay AM, Beck SC, et al. Multilineage potential of adult human mesenchymal stem cells. Science $1999 ; 284(5411)$ : 143-7.

[53] Vater C, Kasten P, Stiehler M. Culture media for the differentiation of mesenchymal stromal cells. Acta Biomater 2010; in press.

[54] Kim DH, Yoo KH, Choi KS, et al. Gene expression profile of cytokine and growth factor during differentiation of bone marrowderived mesenchymal stem cell. Cytokine 2005; 31(2): 119-26.

[55] Kim SW, Kim H, Cho H-J, et al. Human peripheral blood-derived $\mathrm{CD} 31(+)$ cells have robust angiogenic and vasculogenic properties and are effective for treating ischemic vascular disease. J Am Coll Cardiol 2010; 56(7): 593-607.

[56] Medina A, Kilani RT, Carr N, et al. Transdifferentiation of peripheral blood mononuclear cells into epithelial-like cells. Am J Pathol 2007; 171(4): 1140-52.

[57] Badorff C, Brandes RP, Popp R, et al. Transdifferentiation of blood-derived human adult endothelial progenitor cells into functionally active cardiomyocytes. Circulation 2003; 107(7): 1024-32.

[58] Shetty P, Cooper K, Viswanathan C. Comparison of proliferative and multilineage differentiation potentials of cord matrix, cord blood, and bone marrow mesenchymal stem cells. Asian J Transfus Sci 2010; 4(1): 14-24.

[59] Parekh VS, Joglekar MV, Hardikar AA. Differentiation of human umbilical cord blood-derived mononuclear cells to endocrine pancreatic lineage. Differentiation 2009; 78(4): 232-40.

[60] Kogler G, Sensken S, Airey JA, et al. A new human somatic stem cell from placental cord blood with intrinsic pluripotent differentiation potential. J Exp Med 2004; 200(2): 123-35. 
[61] Collas P. Programming differentiation potential in mesenchymal stem cells. Epigenetics 2010; 5(6).

[62] Shapiro AM. Islet transplantation in seven patients with type 1 diabetes mellitus using a glucocorticoid-free immunosuppressive regimen. N Engl J Med 2000; 343(4): 230-8.

[63] Sahu S, Tosh D, Hardikar AA. New sources of beta-cells for treating diabetes. J Endocrinol 2009; 202(1): 13-6.
[64] Gangaram-Panday ST, Faas MM, de Vos P. Towards stem-cell therapy in the endocrine pancreas. Trends Mol Med 2007; 13(4): 164-73.

[65] Motoyama H, Ogawa S, Kubo A. In vitro reprogramming of adult hepatocytes into insulin-producing cells without viral vectors. Biochem Biophys Res Commun 2009; 385(1): 123-8.

[66] Zalzman M, Anker-Kitai L, Efrat S. Differentiation of human liverderived, insulin-producing cells toward the beta-cell phenotype. Diabetes 2005; 54(9): 2568-75.

Received: August 23, 2010

(C) Williams et al.; Licensee Bentham Open.

This is an open access article licensed under the terms of the Creative Commons Attribution Non-Commercial License (http://creativecommons.org/licenses/by$\mathrm{nc} / 3.0 /$ ), which permits unrestricted, non-commercial use, distribution and reproduction in any medium, provided the work is properly cited. 